\title{
Evaluation of Airport Capacity through Agent Based Simulation
}

\author{
Yang Peng ${ }^{1}$, Gao $\mathrm{Wei}^{2}$, Sun Junqing ${ }^{1}$ and Sun Bin ${ }^{1}$ \\ ${ }^{1}$ School of Computer Science and Technology, Tianjin University of Technology, \\ Tianjin 300191, China \\ ${ }^{2}$ Civil Aviation University of China, Tianjin 300300, China \\ pqxx@sina.com
}

\begin{abstract}
The operational capacity of an airport must be dimensioned under a long-term strategic view as its productivity is determined by available infrastructure. To predict the airport capacity and satisfy the domestic airport property, the operation procedure of airport is analyzed from the point of Multi-agent system. The proposed agent based architecture is a microscopic model of aircrafts' movement; each aircraft is represented as an agent capable of getting its routing results from instructing agent, and makes its own deconflicition decisions on the basis of a part of the observable scene local to that aircraft. An example of airport capacity evaluation is given, and the result is verified through comparison with SIMMOD. Finally, further research is applied to analysis the reason for delay.
\end{abstract}

Keywords: Aviation; Airport operations; Capacity Evaluation; System Simulation; Agent

\section{Introduction}

As airports work closer to their maximum capacity, airside airport operations become much harder to deal with. The infrastructure capacity is one of the most important operational and planning parameters of an airport. It is most common to identify airport overall infrastructure capacity with runway system capacity. However, overall airport capacity can also be constrained by ultimate capacity of other infrastructure elements, as well as on how they are functionally related to each other. In order to adapt to the ever-increasing service demand from flights, it is necessary to accurately evaluate the aerodrome operation, especially the operation process in the aerodrome airside via scientific methods.

Results in this study are mainly focused on airport capacity, conducted using an agent based simulation software called AnyLogic, given its capabilities for modeling at a very high level of detail and closely representing reality in terms of airside operations. The model can contribute the development and efficient use of airport capacity as well as effective planning of flight training at airports.

This paper is organized as follows. Section 2 gives an overview of the previous body of related research. The description of the airport's components and the conception of airport capacity are then presented in Section 3 together with the airport surface movement problem. Following this, Section 4 presents the formulation of the architecture of multi-agent simulation system for capacity evaluation, followed by a brief discussion of the routing and deconfliction algorithm. Lastly, a summary of the study's findings is given, along with suggestions for future research at Section 6. 


\section{Literature Review}

\subsection{Airport Simulation for Capacity Evaluation}

Simulation of the airport environment has been increasingly used recently to obtain more realistic estimates of capacity by randomizing the various input parameters. In fact, meteoric improvements in computer technology, especially in the areas of computer graphics; humancomputer interaction and computer networks, have had a significant impact on modeling and simulation. Simulation techniques provide more realistic results for the design and development of airport operations. Among these techniques, discrete-event simulation can provide high level of detail models for the capacity analysis of airports. Numerous tools have been developed based on this technique, such as SIMMOD, CAST, TAAM, RAMS and Airport Machine [1,2]. Using SIMMOD, airside capacity development study is performed an airside capacity development study for Bei Jing new airport. The study proposed a set of procedural and infrastructure developments which offer $2.4-17.1 \%$ increase in airport capacity for different traffic scenarios. SIMMOD was also used to analyze the effect of parallel taxiway configurations on airport capacity. In another study, the relation between air transport movements (ATMs) and runway capacity is studied through commercial simulation tool, and the paper discusses the problem of selecting a suitable peak hour and informs on the annual capacity utilisation of airports worldwide in the form of "traffic ranking curves".[3]

In many applications these commercial simulation tools will satisfy the simulation objectives, but there are examples that the models can't provide the necessary information. Some domestic airports are such examples, which are operated under native rules.

\subsection{Agent in Transportation}

Agent based computing is one of the powerful technologies for the development of distributed complex systems. Traffic and transportation systems consist of many autonomous and intelligent entities, which are distributed over a large area and interact with each other to achieve certain transportation goals. MAS provides a suitable way to model and simulate traffic systems since they offer an intuitive way to describe every autonomous entity on the individual level. In recent years, there is a growing interest in applying agent-based techniques for traffic management [4].

Traffic control and simulation appear to be particularly promising application area for agent technology. In a multi agent traffic-simulation system, each intelligent traffic entity is modeled as an agent. Agents can work cooperatively with each other. MAS has been widely used to investigate traffic-related problems, such as route guidance, urban traffic management and control (UTMC), collaborative driving [5, 6], railway traffic control [7], combined rail/road transport [8], air traffic control $[9,10]$, and the optimization of airport operation [11].

An agent based model enables modeling of every individual throughout the complete simulation lifecycle in complex scenarios, so, to follow these domestic properties, an agent based simulation is created to provide the necessary information for airport ground traffic simulation.

\section{Airside Traffic Control Domain Knowledge}

The airside components of an airport, including airfield geometry, are generally the limiting factor governing the ultimate capacity of the airport. Airside facilities are designed to accommodate the movement of aircraft between final approach and aircraft parking for 
arrivals and from aircraft parking to initial climb-out for departures. Airside components include the runways, the holding bays, the aprons and the gates.

\subsection{Airport Capacity}

Airport capacity generally refers to the ability of an airport to handle a given volume of traffic (demand). There are two commonly used definitions of airfield capacity: 'throughput' and 'practical capacity'. The maximum throughput capacity indicates the average number of movements that can be performed on the runway system in one hour in the presence of continuous demand, while adhering to all the separation requirements imposed by the air traffic management (ATM) system. Practical capacity is the number of operations (takeoffs and landings) that can be accommodated with no more than a given amount of delay, usually expressed in terms of maximum acceptable average delay. The practical capacity is equal to 80 or 90 percent of the maximum throughput capacity.

\subsection{Taxi Process and Taxi Conflict}

The most important stage of capacity evaluation simulation is the analysis of traveling times and delays for the selected airport. According to ICAO Doc.9830, aircraft taxi collision conflict detection and resolution need to meet three requirements:

(1) Providing conflict alert mechanism of two levels (include collision conflict pre-alerting and warning, respectively); (2) Ensuring air traffic controllers and pilots have enough response time; (3) The disturbance of false alarm to normal work of air traffic controllers and pilots should be avoided, and also conflict resolution measures should not introduce new conflicts.

The taxi process of flights is a major part of present and future airport surface operations, which encompasses an aircraft's movement from a parking position to an entry point of the departure runway or vice versa from a runway exit to a parking position.

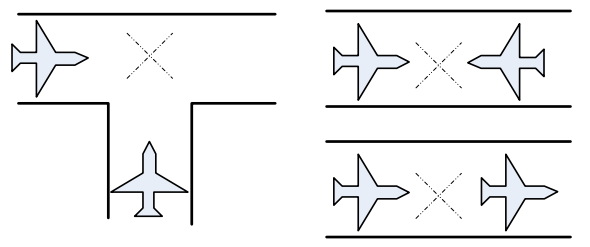

\section{Figure 1. Typical Taxiway Conflicts}

Conflicts appear when different flights try to use the same edge or crossings of edges at the same time. Figure 1 present the typical taxiway conflicts at the airport surface. On most of China airports, controlling airport surface traffic is done by controllers in manual way. Therefore, we have to intermingle the human factors on aircrafts ground movement decision through the simulation model.

\section{Model Description}

In this study, an airport system is modeled using AnyLogic agent based simulation tool with a fine level of detail. Several assumptions were made in order to increase the flexibility and ease of use of the model, while maintaining the integrity and accuracy of the results. Some of the major assumptions used in the model are listed below. 
(1) No simultaneous aircraft occupancy is allowed for the runway and final approach path; (2) Safe separation minima between aircraft are determined according to relevant separation standards [ICAO (2007)]. (3) Aircraft traffic mix is restricted to general aviation category aircraft. (4) Landing and take-off operation is performed in one-direction. (5) The departure sequencing strategy is set as a First In First Out (FIFO) strategy.

\subsection{Architecture of Multi-Agent Simulation System}

The developed model considers each subsystem as a type of agent that acts based on its local knowledge and its interaction with other agents. For design of Multi-agent based airport simulation system, we created the following pseudo-agent class, as described in Figure 2.

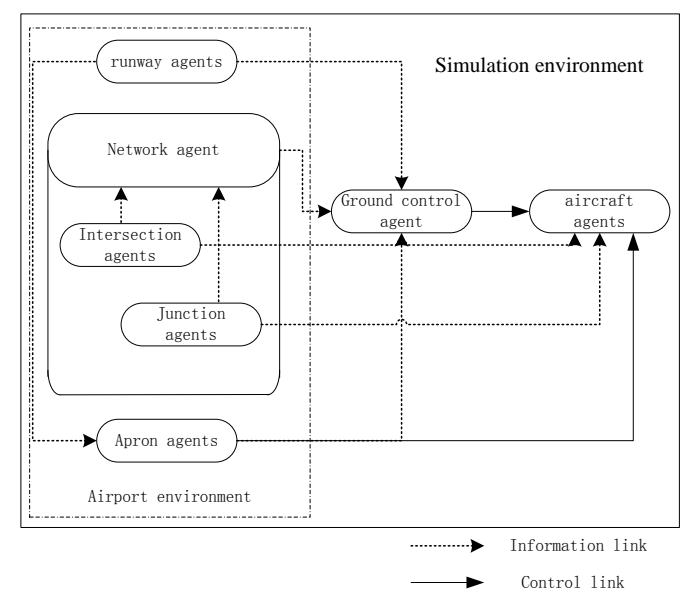

Figure 2. Relationships between Agents

1) Aircraft agent class (A-agent class); each agent of this agent class simulates and monitors a particular aircraft at the airport;

2) Network agent class ( $\mathrm{N}$-agent class); this agent store the traffic inform of the airport traffic network system, and the agent may assists the aircrafts to make route decision;

3) Junction agent class (J-agent class); each agent of this class assists the network agent to update the particular junction traffic information(for example the direction of the junction);

4) Interaction agent class (I-agent class); each agent of this class assists the aircraft agents to the nearby traffic information while;

5) Ground control agent class(GC-agent class); this agent provide the initial routing results for the A-agent, but while the A-agent is conflicted with other agents, the Aagents can deconflict the problem through peer-wised cooperation;

6) Runway agent class(RW-agent class); each agent of this class is applied to simulate the runway and it was afforded statistical methods to recorder the utilization rate of the runway;

7) Apron agent class(AA-agent class); Two different roles are assigned to the AA-agent class. First, store the traffic and aircrafts information at the apron, second, control the take off process of aircrafts that parking at the apron. 
Simulation environment plays here the role of an active program entity. It is intended for simulation and visualization of real time situation at the airport. It initiates real time events reflecting the results of operation of entities involved in airport ground traffic and traffic control.

\subsection{Organization of Information Exchange}

Autonomous behavior of an aircraft in constrained environment, i.e., the airport, assumes that each aircraft has to possess the information on current positions, courses and anticipated movement plans of other aircrafts operating within the airport traffic network, at least those that potentially may violate the separation standards. In the proposed multi agent system, this information is gathered by A-agent, J-agent and I-agent. A-agent not only registers its information to the junction that it's currently located on, but also to the I-agents that are connected to the very junction.

Since most junctions propose two intersection and most intersections have more than one junction, so each aircraft will belong to several groups depending on its current air traffic situation. That is why aircraft groups may be overlapping.

To further decrease the communication overhead, every A-agent, at any time instant $t$, takes into account potential conflicts within two groups, namely the intersection of its current location and the next one it plans to transit to. Thus, in the developed approach, to compute its own conflict-free behavior, the A-agent relies upon information exchange with aircrafts of no more than two groups determined on P2P interaction basis. Table 1 shows the aircraft's data that need to be exchange.

Table 1. Some Information to Exchange among Agents

\begin{tabular}{|c|c|}
\hline \multicolumn{2}{|c|}{ Aircraft's related data } \\
\hline Aircraft & Aircraft's identifier \\
\hline Current Position & ID of Junction and Intersection in which aircraft is currently located \\
\hline Current State & Being blocked or on the move \\
\hline Next Intersection & ID of Intersection into which the aircraft has to overcome next \\
\hline
\end{tabular}

\subsection{Routing Algorithm}

Airport surface traffic networks can potentially be quite large, so it is not feasible to make a global optimal routing solution with the infrastructure as a whole. Instead, we assume that the A-agents are routed sequentially. When an A-agent is ready, it has to be routed respecting previous reservations by other A-agent using the taxiways. The routes which have been previously calculated will be registered to the $\mathrm{N}$-agent, and do not change as new aircraft are taken into consideration.

The objective for each of the sequential routings is to find the routing with minimal taxi time among all remaining routings. The approach described here is based on research by Gawrilow et al., [12], but further modified. The quickest path problem with time windows and predefined junctions (QPPTWPJ) algorithm is a generalized vertex-based label-setting algorithm based on Dijkstra's algorithm and can sequentially route aircraft on the airport surface. The predefined junctions are applied to guarantee that the routing algorithm satisfied the control habits.

\subsection{Deconfliction Algorithm}

Although we have created the QPPTWPJ algorithm, but A-agents may still conflicted with each other during the movement for stochastic perturbation. So we have to design a 
deconfliction algorithm. An A-agent is able to communicate with the intersections of its current junction, and the I-agent is able to communicate with the nearby I-agents, so in this way the A-agent is capable of find the potential conflicts with other A-agents. Figure 3 illustrates the communication ranges of A-agent and I-agent.

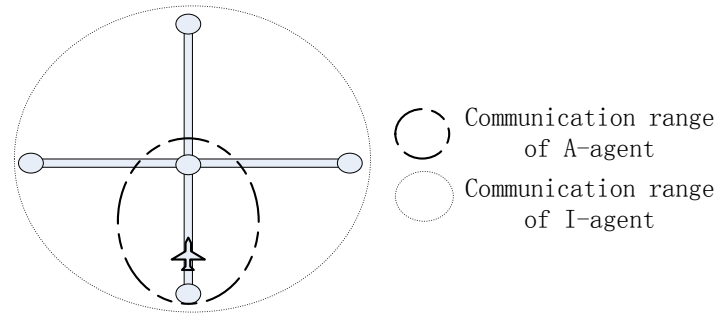

Figure 3. Communication Range

To reduce the complexity of the distributed deconfliction task, the algorithm is performed in two steps. At the first step, every A-agent computes its priorities regarding to all peers at its communication range. At the second step, the A-agent of the highest priority is automatically "granted" permission to use the junction or intersection "resources" according to its current plan. Figure 4 is the message sequence diagram of A-agent while it tries to access forthcoming intersection.

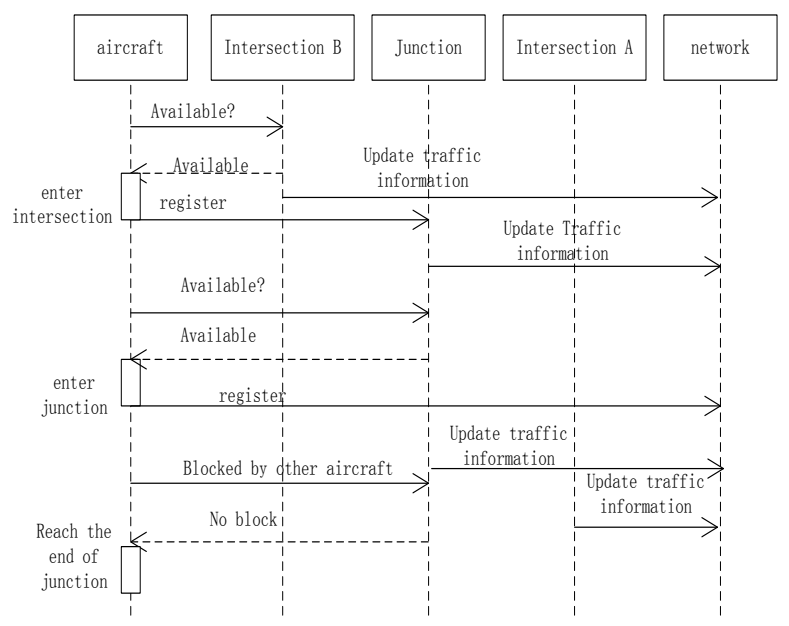

Figure 4. Message Sequence Diagram

It is worth to note that in practice more rules and more aircraft attributes have to be used in the deconfliction process. But this task is out of the paper scope so far.

\section{Model Development and Cases Study}

\subsection{Simulation Environment and Data Collection}

The target aerodrome is configured with two parallel runways which can meet the land/takeoff service demand from various aircraft types. At any time, the parallel runways are simultaneously available for departure and arrival operations. 
Separation matrices for taxing control must be applied during the operation of the airport, as is listed in Table 2, these separation minima are used in our model.

Table 2. Aircraft Separation Minima

\begin{tabular}{ccccc}
\hline & \multicolumn{2}{c}{$\begin{array}{c}\text { Arrival-Arrival } \\
\text { (nautical miles) }\end{array}$} & \multicolumn{2}{c}{$\begin{array}{c}\text { Departure-Departure } \\
\text { (Seconds) }\end{array}$} \\
\hline Leader & Heavy & Large & Heavy & Large \\
Heavy & 4 & 5 & 120 & 120 \\
Large & 2.5 & 2.5 & 60 & 60 \\
\hline
\end{tabular}

\subsection{Model Verification}

Validation of the agent based model is a critical step for simulation. Being one of the oldest and most commonly used air traffic simulation modeling tools, SIMMOD outputs can be were used as the baseline for expected results. Multiple replications of the models are run, with the system and all statistics reset between replications. The average delay of aircrafts is used to measure the quality of a scenario after execution and to compare.

Case 1: In this scenario, the air traffic simulation flow of the aircraft amount ranged from 50 sorties per hour to 70 sorties per hour and the ratio between arrival and departure amount is one-to-one. Using the average delay, the agent based model was compared to SIMMOD.

As shown in Figure 5. The red curve is the distribution of average aircraft delay for increase of traffic volume, estimated by the agent based simulation model. The blue curve is the distribution of average aircraft delay for increase of traffic volume generated by the simulation of SIMMOD. As can be seen, they agree well.

Case 2: The sequencing of aircraft in the airspace needs to be seen as an important influence on the airside capacity and performance of an airport system. The distribution of heavy aircraft to total aircraft category is defined as Mix-Index, and the Mix-Index of arrival aircraft needs to be regarded as an important influence on the airside capacity and performance of an airport system. Suppose that the tolerable level of average delay is 4 minutes, and the corresponding capacity is regarded as runway practical capacity. Figure 6 presents the effect of Mix-Index on airport practical capacity.
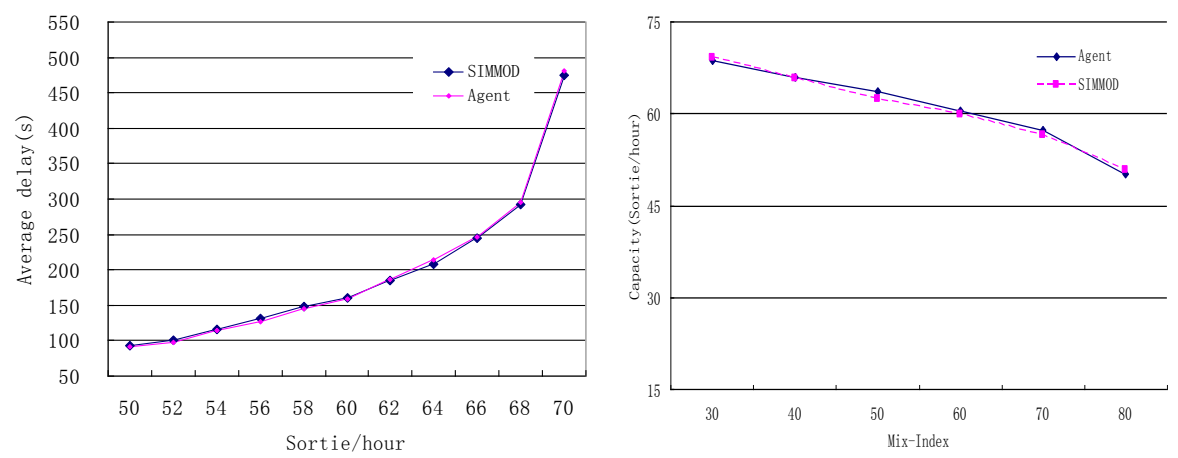

Figure 5. Delay and Demands Figure 6. Mix-Index and Capacity

From the Figure 6, we can see that the capacity decreases as the Mix-Index decreases. As can be seen, the range over which the distributions extend match well in all the sets of streams. The distributions themselves match very well on the long-side. The distribution of practical 
capacity estimated by the agent based simulation is in reasonably good agreement with baseline results.

By inspection of the confidence intervals to the above cases, we can find that the results of the agent based models are not different with that of SIMMOD.

\subsection{Further Analysis}

Since the macroscopic model does not offer the necessary level of detail to analysis the reason for delay, in this part, we will apply the agent based model to further the research.

As can be seen from Figure 7, the flight delay increases as the traffic volume increases and the departure delay accounts for a large part in the overall delay. With the increase of traffic volume, the percentage of on time departure aircrafts decrease drastically, while it only has moderate effect on the on time percentage of arrival aircrafts.

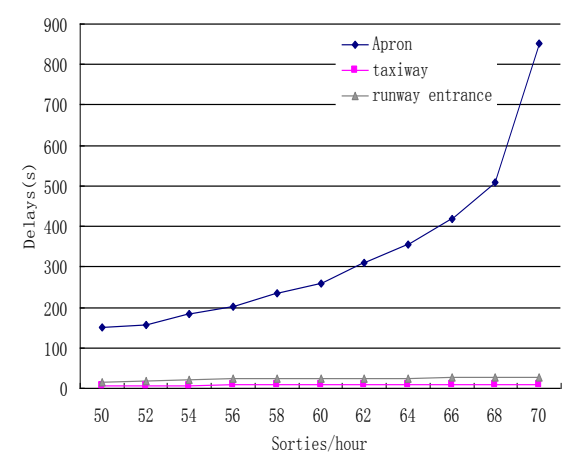

Figure 7. Comparison of on Time
Percentage

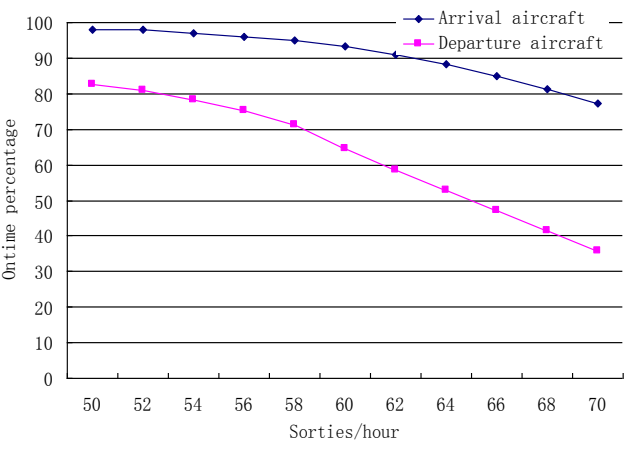

Figure 8. Delays at each Subsystem

As can be seen from Figure 8, the delay at apron accounts for a big part of the departure delay, whereas the taxiing and the entrance holding processes do not have much effect on the departure delay. The reason for that is the departure aircraft has to spend extra time at apron for departure instruction. Therefore, we can conclude that current apron and taxiway operation in the airport could meet the traffic service demand and the bottleneck of airside operation mainly lies in the parallel runways.

\section{Conclusions}

This research has provided an approach to estimate the performance measures of an airport terminal's landside processing facilities. The proposed simulation model that seeks aircrafts and traffic resources using individual agents that attempt to fulfill a specific objective is developed. The coordination between agents is achieved through protocols. The hierarchical architecture makes it possible to give microscopic model of aircrafts' movement, and intermingle the human factors on aircrafts' movement decision.

In the present work we have created an agent based simulation model, but there is still much need to be done for improvement. Some possible extensions are integrating the traffic simulation within airport airspace, and intermingling more habits orientated controls to the movement of the aircraft. 


\section{Acknowledgements}

This work is financially supported by National Nature Science Foundation of China under the Grants 61039001, and National Science technology Support Plan Projects under the Grants 2011BAH24B10.

\section{References}

[1] T. Wang and W. Gao, "Runway Layout Comparison of Beijing New Airport", Computer systems \& application, vol. 23, (2014).

[2] M. Bazargan, K. Fleming and P. Subramanian, "A Simulation Study To Investigate Runway Capacity Using TAAM", Proceedings Of The 2002 Winter Simulation Conference, (2002) December 8-11.

[3] D. Wilken, P. Berster and M. C. Gelhausen, "New empirical evidence on airport capacity utilisation: Relationships between hourly and annual air traffic volumes", Research in Transportation Business \& Management, vol. 1, (2011).

[4] B. Chen, H. H. Cheng and J. Palenc, "Integrating mobile agent technology with multi-agent systems for distributed traffic detection and management systems", Transportation Research Part C: Emerging Technologies. vol. 17, (2009).

[5] M. Jeon, A. Riener, Ju-Hwan Lee, J. Schuett and B. N. Walker, Cross-cultural differences in the use of invehicle technologies and vehicle area network, Proceedings of the 4th International Conference on Automotive User Interfaces and Interactive Vehicular Applications, (2011) October 17-19, New York, USA.

[6] S. Halle and B. Chaib-Draa, A collaborative driving system based on multiagent modelling and simulations. Transp. Res. Part C: Emerging Technol., vol. 13, (2005).

[7] D. Zhao, Y. Dai and Z. Zhang, "Computational Intelligence in Urban Traffic Signal Control: A Survey, Systems, Man, and Cybernetics, Part C: Applications and Reviews", IEEE Transactions, vol. 42, (2012).

[8] L. M. Gambardella, A. E. Rizzoli and P. Funk, "Agent-based planning and simulation of combined rail/road transport”, Simulation-Trans. Soc. Model. Simul. Int., vol. 78, (2002).

[9] B. Chen and H. H. Cheng, "A Review of the Applications of Agent Technology in Traffic and Transportation Systems, IEEE Transactions on Intelligent Transportation Systems, vol. 11, (2010).

[10] W. G. Li, M. V. Pinheiro, and A. C. M. de Melo, Method to balance the communication among multi-agents in real time trafflc synchronization, Proceeding of Fuzzy System Knowledge Discovery, vol. 36, (2005).

[11] J. Tang, S. Alam, H. Abbass, and C. Lokan, Modelling and evolutionary multi-objective evaluation of interdependencies and work processes in airport operations. Proceeding of 1st ACM/SIGEVO Summit GEC, (2009) Shanghai, China.

[12] E. Gawrilow, E. K"ohler, R. H. M"ohring, B. Stenzel, "Dynamic routing of automated guided vehicles in real-time" Mathematics - key technology for the future, Springer Berlin Heidelberg, pp. 165-178, (2008). 
International Journal of Grid Distribution Computing Vol.7, No.6 (2014) 